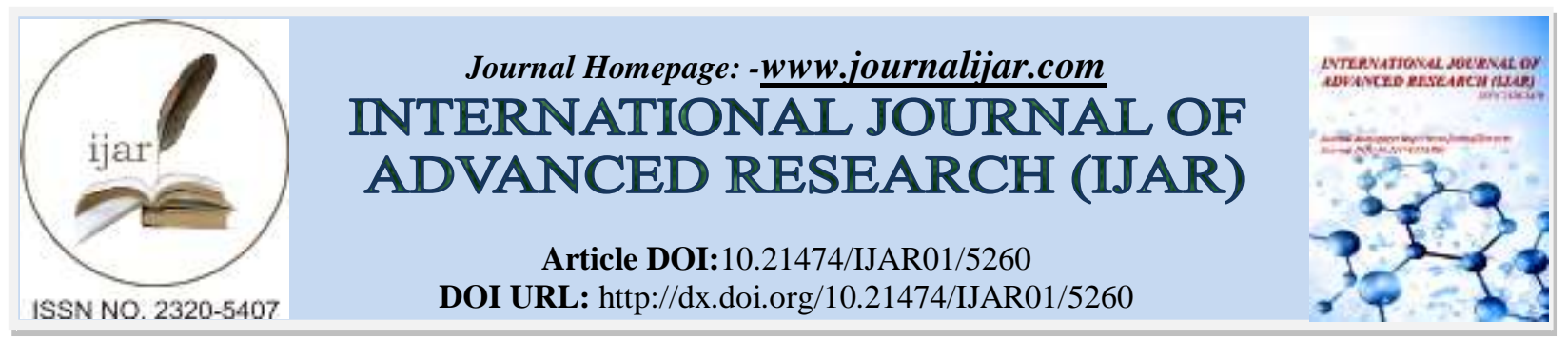

RESEARCH ARTICLE

\title{
SUBMISSION OF NEW MANUSCRIPT FOR EVALUATION.
}

\author{
Dr. Mohammad alajmi. \\ Ministry of health - kuwait.
}

\section{Manuscript Info}

Manuscript History

Received: 23 June 2017

Final Accepted: 25 July 2017

Published: August 2017

Keywords:-

Neuroplasticity; Synapses;

Angiogenesis; Rehabilitation; Brain injury.

\section{Abstract}

Acquire brain injury is a serious neurological complication that occurs after the birth of an individual. It could be traumatic and non-traumatic. Traumatic cases occur due to accidents, injuries, falls whereas nontraumatic cases occur due to stroke, tumor, alcohol consumption, etc. The damage induced resulting from the injury could be moderate or severe and affects the individual's cognitive and behavioral skills. There is a need of recovery to get restored to normalcy. Neuroplasticity process has been considered vital in offering recovery. The main objective of the paper is to provide a description on the neuroplastic changes following acquired brain injury. A literature search was carried out by using databases like Pubmed. Pertinent articles were retrieved. It was found that neuroplasticity is a sequence of events that necessitate the input of various internal and external recovery mechanisms. These mechanisms in turn involve both recovery and compensation. Recovery is intended for the restoration of the lost functions while compensation is intended for the acquisition of novel behaviors to substitute the ones lost following the injury. During the recovery, neurogenesis and angiogenesis tend to work in an integrated manner. Apart from this, training induced recovery like that offered in a rehabilitation setting was also proved to be instrumental in inducing neuroplastic changes. Tremendous research was done with the aid of rat models to assess the neuroplasticity. The ultimate objective of these recovery modes is to ensure improved neuroplasticity such that there will be increased axonal growth, synaptic connections, signal transmissions in the brain and also sense perception to the damaged limbs. The individual could be able to perform daily living activities independently.

Copy Right, IJAR, 2017,. All rights reserved.

\section{Introduction:-}

Living organisms in the universe possess an adapting characteristic for their surroundings. This is essential for them to struggle for existence and survive. Most importantly, it is the intellectual ability of the brain that judges and guides the organisms. This process that is continuous and predetermined relies, in fact, on certain structural rearrangements in brain. These arrangements enable the organisms to withstand against adverse environmental conditions. So, it could give a sense that certain neural networks have influenced such changes. In such context, the present description is concerned with highlighting about "Neuroplasticity after acquired brain injury". Briefly, plasticity refers to the property of a substance or material which could be easily shaped or moulded in various forms. 
In the biological context, it refers to the organisms' adaptability towards environmental variations between in habitats of several kind (Plasticity,2014). Similarly, neuroplasticity is the capability of the brain to self-reorganize by forming novel neural networks during life (Neural plasticity,2014). It facilitates the nerve cells to sustain the injuries or illnesses and get adjusted to encounter novel environmental tasks. Here, the reorganization of brain could occur by axonal sprouting mechanism where damage resistant (undestroyed) axons form novel endings of nerves to establish a reconnection with neurons that possess injured connecting ends. Undestroyed axons could also lead to the formation of nerve endings and establish a connection with other undestroyed neuronal cells. As a result, novel neural pathways could be formed that take up a required functional activity. For instance, if there is a damage of one hemisphere, another hemisphere could acquire few of its roles. Thus, there will be a compensation for damage via reorganization and novel connection formation (Neural plasticity,2014).

Neuroplasticity after acquired brain injury (ABI) is the area of much research interest. Acquired brain injury (ABI) constitutes a brain damage that takes place after the individual is born. Say, that damage could be from a severe blow to head, oxygen deficiency, disease or infection.ABI IS of two types. These are Non-traumatic and traumatic. Non-Traumatic ABI occurs due to cases of non- violent nature. These are stroke, hypoxia, brain aneurysm, brain tumors, anoxia,toxic injury, infection and drug or alcohol abuse. Traumatic ABI occurs due to sports related injuries, falls, open head injuries, assaults, road accidents (Acquired brain injury,2014).

Next, brain has a special important function of memory. It recalls the immediate events that occur in nature via short term memory. But, these memory events are later grouped together into long term memory for the purpose of later recall. This process known as memory consolidation needs synaptic plasticity that involves gene expression changes and physical changes in synapses of neurons. Studies on synaptic plasticity reported that immediate -early genes (IEGs) could change the expression following neuronal events. These IEGs facilitate a process called potentiation (LTP) that improves the connections of synapses and gathers memory events. But, since every event could not turn into a long term memory, the synaptic remodeling response that works in the opposite fashion, long-term depression (LTD) influence synaptic plasticity. Thus, variations in the gene expression linked with LTD and LTP contribute to physical variations in the neuronal synapse that could increase or suppress connections of synapses. Further, in the postsynaptic density, the expression of several important neuronal receptors is also instrumental to memory and learning (Synaptic plasticity,2013).

So, alterations in gene expression occur and make a short term memory become long term memory in both vertebrates and invertebrates. In the synaptic plasticity process, neurons have the potential to change communications among them through connections of synapses. This could occur in response to certain neurotrophic factors or electrical stimulation patterns (Ritcher \& Klann,2009).Next, brain exhibits remarkable reaction to direct and indirect tissue damages.

A direct tissue damage could occur from outside, for instance, due to a direct blow to the head. An indirect tissue damage could occur from inside, for instance, due to an injury resulting due to the brain movement within the skull. This leads to axonal, and blood vessel injuries from shearing forces as the head speed up and retards following the impact of injury(Head Injury, 2011). In response to the injuries, the reaction of the brain could be considered as a complex process. In the initial weeks following a brain injury, there could be swelling, blood seepage or variations in brain's chemical circuitry (Novack and Bushnik,n.d.). As a result, the brain loses its normal hygienic functional activity. When the swelling subsides, and blood circulation normalizes, there will be a restoration of brain function. Most importantly, these changes reflecting an early recovery are grouped under the following terms.

Coma: Here, the individual is unconscious, fails to respond to sounds or visual stimuli, communication, and displays emotional responses of poor degree. Vegetative State: Here, the sleep-wake cycles are normal, and the person slowly gets oriented to sounds and visual stimuli. Minimally Conscious State: Here, the person is conscious partly and knows the sources of sounds and visual stimuli, approaches for things, reacts to commands, speaks occasionally and displays emotion. Following, traumatic brain injury (TBI), there will be confusion and disorientation. There will be a loss of ability to attention and learning, and restlessness, nervousness, frustration and aggressiveness. The person could become hyperactive to stimuli (Novack and Bushnik,n.d.).

According to researchers, there are two phases of brain injuries (Ryest,2014). These are phase 1 and phase 2 .

Phase 1. In this phase, tissue injuries are of direct type that is accompanied with altered cerebral blood flow and altered activity in the metabolism. This results in edema formation and cytoarchitecture variations like membrane 
permeability. Subsequently, a series of pathological events get initiated. Phase 2. Certain inertial forces lead to brain tissue compression whereas, contact forces lead to hemorrhage, contusion and laceration. Such forces result in diffuse axonal injuries. This could affect blood vessels, axons,junctions between the frontal and parietal lobes, junctions between white and gray matter, and the corpus callosum. Due to this, a series of pathological processes gets initiated.

\section{Phase 2:-}

In this phase, a disruption of neurons occurs that results in depolarization and excitatory neurotransmitter's release. This also enables the release of sodium and calcium ions that lead to intracellular damage. Then chaplains and caspases get released that contribute to cell death. Here, calpain's release contributes to necrosis where cell death occurs in response to defects in metabolism and hypoxic processes. As a result, inflammatory responses develop and cause the removal of cells. Similarly, caspase release begins the apoptosis process where intact cells contribute to cell transport disruption, cell membrane disorientation and cell demise. During the processes of injury, bloodbrain barrier (BBB) also occurs. This could be again a primary direct injury and secondary damage to the endothelium of the BBB (Ryest, 2014).

So, after brain injury, neurons, glial cells such as oligodendrocytes, microglia, astrocytes and cells that synthesize cerebrospinal fluid (CSF) and provide wiring to brain's ventricles get affected.

Due to this, the synthesis, distribution and CSF reabsorption gets affected, and variations in the metabolism and cell function occur. This cell injury occurs both at the vicinity and distant areas of damaged brain regions.

A brain consists of six parts that could be affected when a head injury occurs. The parts are brain stem, cerebellum, frontal lobe, parietal lobe, occipital lobe, and temporal lobe.

In certain cases, a single area gets affected, whereas, in the majority of cases, multiples area get affected. Each part of the brain has a specific function. When different parts of the brain get affected, there could a functional decline of that specific part. This will depend on the magnitude of the damage to that part. There could be physical problems, cognitive problems and behavioral problems.

Physical problems: In many cases, persons with TBI are flexible in using their arms and feet. But in the long term, there will be a reduction in the coordination and balance.

Cognitive problems: Here, functions like concentration, attention, memorizing new events get affected. They will be slow in thinking, speaking and solving problems. They continue thinking on a task for a long time and fail to switch to separate tasks when they encounter problems. Speech and language problems are present.

Behavioral problems: These issues result due to several factors. Initially, the changes are driven from direct damage to a particular brain tissue, say frontal lobe. This part is for regulating behavior and emotions. Cognitive problems contribute to emotional disturbances. Say, an individual who fail to concentrate on a conversation could express frustration and upset mental attitude.

Similarly, major life changes that occur in such people could also leave them in potential emotional behaviorism. Say, an income loss or employment failure and family role variations, could contribute to depression and frustration (Novack and Bushnik,n.d.).

\section{Recovery:-}

A recovery process following the brain injury relies on Plasticity (Elovic,Baerga \& Cuccurullo,2013). This is in turn affected by environment, stimulation complexity, task repletion and motivation. The recovery mechanisms are: a) Neuronal regeneration and b) Unmasking neural reorganization.

Neuronal Regeneration: Here, intact axons form a synaptic connection via axonal and dendritic sprouting at the site of damage. This could lead to recovery or no effect or unneeded symptoms. The total period of such recovery could last up to months following the injury (Elovic,Baerga \& Cuccurullo,2013). 
In connection with the brain repair concept, scientists working on neural interface systems have proposed a hypothesis (Guggenmos et al., 2013). This is that a neural prosthesis could enable recovery that plays a role as a communicating link between distantly located sites in the cerebral cortex of the brain.

To test this hypothesis, scientists have employed a rat model and injured the primary motor area. This disrupted the communication between somatosensory and motor areas and contributed to altered grasping functions. But following a microelectrode implantation in the cerebral cortex, neural prosthesis was capable of stimulating electrical stimulation in the somatosensory cortex and improved functional connectivity between the targeted zones. This indicated that impaired neural pathways could get functionally restoration either through plasticity or experimental stimulation (Guggenmos et al., 2013).

Further, the neural prosthesis was also found to restore behavior functions in individuals with traumatic brain injury. Rat models that received a two week prosthesis implantation were able to approach and obtain a food pellet. Rats exposed to several stimuli were able to get low proportion of the pellet. Those with no exposure to any stimuli received a much lesser proportion than the exposed ones. This indicated that the sensory stimulation plays a role in the restoration of movements after brain injury.

Similarly, movement restoration could occur through neural reinnervation strategy. This is because it provides a chance to evaluate the effects of cortical plasticity when behaviorally vital sensory afferents are re-guided to another body part from their original location (Marasco and Kuiken,2010).

Scientists worked on a model where damaged cerebellum was treated with peptide known as Brain Derived Neurotrophic Factor (BDNF) (Guggenmos et al., 2013). This protein is important for the function of the neuronal pathway that connects the stem with the cerebellum.

As a result, scientists demonstrated that the new axon terminals interacted with uninjured neuronal cell network to restore spatial orientation and synchronized movement.

In the present case, the researchers have extended the use of this model and showed that the terminals of new axons interact with the network of undamaged neuronal cells to restore their associated functions, such as synchronized movement and spatial orientation (Guggenmos et al., 2013).

Earlier, it was described that restoration of functions could be accomplished via functional system reorganization .This is either a center substitution in the opposite hemisphere or a similar component reorganization of the functional system that does not involve in the injury. Here, the technique of psychological reorganization plays a vital role (Stern, 1964).

In the recent period, vision restoration was also being considered. This is because vision uses several cognitive mechanisms. To say, via several pathways visual information transfers to the regions of the cortex. This is nothing but visual neuroplasticity and this could improve visual activity (Sabel, Kenkel \& Kasten,2005).

Further, the impact of brain injury on the abilities of an individual could require a process of services and rehabilitation (The brain injury,.n.d.). This is much associated with the injury nature and its time when the injury occurred. To say, in order to get restored to the prior activities, an injured brain could rely on physical therapy, say for instance a range of motion therapies. A range of methods is implemented from the perspective of the injured persons' needful daily living activities. These are weight bearing, tilt table, sitting balance,exercise balls, gait training, strength training, pedalers, and speech therapy (The brain injury,.n.d.).

These approaches appear to be of great help to patients who possess a range of complications. These are that they i) have minimum consciousness, ii) are in need of their head and trunk control and iii) able to achieve more strength and body coordination.

Added to this, occupational therapy could also enable an individual to perform the activities of daily living. With this therapy effect, an individual could regain previous abilities and assists in achieving freedom in all walks of their life. For this purpose, the occupational therapist utilizes every household items such as forks, spoons, tooth brushes, hair brushes, to assist the patient relearn the required skills. 
If a weakness is identified, there could be the usage of tools and exercise programs identical to physical therapy (The brain injury,.n.d.). So, activity recovery following a brain injury much relies on both physical and occupational therapy that enable the brain to perceive the sensory information.

Later, neurobiological changes during recovery are important to consider.

Initially, neural circuit recruitment is important. This is because brain injury disturbs neuronal circuitry leading to the death of glial cells and neurons thus impairing the links between them. This involves extensions of axons and dendrites through which signal processing occurs by neurotransmitters. So, a brain injury frequently causes the deposition of neurotransmitters in excess in the brain tissue, especially glutamate that could overexcite neurons and causes their death (Pekna \& Pekny,2012).

The neuroplasticity involved here is that there could be variations to synapses like synaptic plasticity and synaptogenesis, dendrite variations like high arborization spine and dendritic growth.

Others include axonal sprouting, neurogenesis in particular brain regions such as specific subgranular zone of hippocampus and subventricular zone of some areas, perinfarcted areas and substantianigra. These changes are mainly intended for contributing to increase in neurons or synapses or strengthening the synaptic strength

(Reyst,n.d).

Neural recruitment during injury is believed to be much feasible with glial cells. These constitute microglia, oligodendrocytes and astrocytes. For instance, astrocytes could synthesize nutrients and could help in cell viability. They have a protective effect during damage, induce brain homeostasis and offer neuron nutrition and neurotransmitter recycling. Especially, they are instrumental for regeneration and plasticity and neuronal synapse generation and their functional regulation (Pekna \& Pekny,2012).

Researchers reported that glial cells could be reprogrammed into functional neurons.

This was made feasible with Neuro D1, a neural transcription factor that was subjected to a retroviral expression system. This factor plays a vital role in reprogramming NG2 cells into GABAergic and glutamatergic neurons. So, reactive glial cells offer an advantage by recruiting functional neurons for repairing an injured brain (Guo et al.,2014).

Further, training of new movement sequences relies on rehabilitation approaches (Wilson et al., 2014). This method could use virtual environments. Virtual reality (VR) systems facilitate the presentation of environment training in a much reliable and automated manner. This allows movement analysis at three behavior levels. These include 1) neurocognitive performance 2) patterns and forms of movement that reflect the patient's movement at a specific recovery stage, 3 ) functional result of movement.

The success of level 1 depends on the use of internal models for action by the patients and the speed at which the skills learned in a rehabilitation setting is generalized for the real life scenarios. Among the neurocognitive models of motor function, internal modeling is very essential. Internal models are of two types. Forward models use motor commands in predicting the movement of limbs in the future. Inverse models use commands to attain a specific objective. For instance, in body -centered coordinates, intraparietal sacculus (IS) neurons have a vital role in coding a target position (Wilson et al., 2014).

Based on internal modeling, researchers have devised motor imagery training protocol by using an interactive CD ROM. This is intended for movement education uses verbal scripts and digitised models to stimulate the performer about the effective movement. Researchers have induced external memory prompts in a goal directed action. They found enhanced functional outcome on variety of everyday activities including those performed previously. So, for rehabilitation movement, this model has good implications. This could convert the performance of client from slowly controlled mode to fast, automatic, and time saving mode. 
Level 2 Recovering patients could adopt movement form and efficiency. Such patients could undergo a 'movement form' transitions that reflect control parameter variations such as flexibility, tone and strength. This is nothing but movement kinematics (Wilson et al., 2014).

In the rehabilitation setting, functional assessment is the main focus. Say, goal-oriented acts to certain useful objects were reported to shoe flexible and rapid reaching movements. For people with brain injury, the performance is much associated with several biochemical constraints. These are decreased coupling between shoulder and elbow, decreased agonist strength, high muscle tone due to spasticity. But this mode of recovery could get prevented if the patient fails to adhere to a specific therapeutic regimen. The tasks presented to the patient must occur in a meaningful and stimulating manner. So, virtual reality has proven ability in inducing balance and mobility skills in the patient. Researchers have been coming up with novel activity methods to speed up the recovery following brain injury. On these grounds, in the rehabilitation settings, the activity of coffee preparation has drawn much attention This is because coffee was believed to possess an ongoing presence, it's making or the problems encountered while making are considered as brain injury symptoms.

Making coffee could reflect a failure in planning a sequence of complex movements that are required to accomplish a task that has many stages. Similarly, recreation is another novel way of training for individuals who fail to involve in a traditional rehabilitation and are incapable of resuming their work.

This could be accompanied with a meaningful socialization or community integration.

Activity training better prepares the individuals to stay independently in the community.

This could be accomplished by considering community and family members as paraprofessionals and offering them training, peer support programs, home-based rehabilitation, social activities, workplace, and association with the organizations of community and enhancing the awareness about brain injury.

According to the available evidence, following a brain injury, the brain regions stop obtaining sensory information through afferent nerves and the dendritic nerve gets retracted. As a result, synaptic loss occurs with several neurons. This response is a maladaptive response. In contrary, there will be an increase in the dendritic arbors in non-affected brain regions following injury. This adaptive response of brain is to enhance synapses in intact areas and this finally offers a cortical rewiring.

Next, following brain injury, a sprouting and reorganization of axons occurs. Sprouting refers to an adaptive response where a high axonal development results in higher synaptic levels after reinnervation process. But, glial scars could prevent axons from translocating to the targets. It was also found that in cases of damaged hemisphere, variations related to topographical maps occur in motor areas where several areas regulating motor movements provide compensation for the destroyed areas. So, synaptic variations are considered as the neurobiological basis of motor map variations. This involves synaptogenesis where novel synapses et developed via synaptic plasticity, axonal sprouting, and dendritic growth. With this process, existing synapses become firmly established via longterm potentiation.

Angiogenesis: This constitutes the formation of novel blood vessels from the pre-existing ones. For instance, a neuronal death is caused when blood flow gets stopped in a condition, ischemic stroke. The advantage is that blood flows back to earlier damaged areas and helps in promoting metabolic activities. In a study, researchers assessed the angiogenesis role while studying neurovascular response following stroke. Here, a neurovascular damage was able to contribute to blood brain barrier disruption in the acute phase of injury. But in the delayed phase, neurogenesis and angiogenesis, serve as initial responses following a stroke episode. In angiogenesis process, the cytokine that is more associated is vascular endothelial growth factor (VEGF).

This hormone offers neuroprotection and is essential for post-injury recovery. A blockage of VEGF receptors and prevention of their upregulation could lead to a low vascular proliferation. Such a reduction in positive vascular variations was reported to influence angiogenesis. Excitation: In this process following an injury, variations in the excitability of the intact and destroyed hemispheres could affect the function of cortical regions. Across hemispheres, excitory changes could occur rapidly. To this end, researchers have proposed the 'interhemispheric rivalry' according to which variations in the excitability of identical regions takes place between specific motor 
areas of hemisphere. For instance, neuron inhibition, also known as hyperpolarization, occurs in the damaged hemisphere while neuron excitation takes place in the intact hemisphere. Following stroke in the chronic phase, a recovery could be more on the damaged side and less on the intact hemisphere. Such an activation shift on the unaffected side is "the sign of a distressed system". If there is a much involvement of the damaged site, there could be a better result in the recovery. But, if the patient needs to depend on the unaffected area for functional activity, there could be a poor result in with regard to the recovery. The role of an impaired preforontal cortex after a damage is such that it compensates for the damaged areas of PFC in the opposite hemisphere. These excitory events following injury are identical to neuroplastic properties in the intact brain. So, depending on the challenge levels a damaged hemisphere need to encounter, this mode of compensation works. In rat models, the excitory behavior of synpaptc connections was addressed (Jin, Prince and Huguenard, 2006).It was reported that dentate gyrus granule cells and sprouting axons of pyramidal neurons contribute to recurrent excitory synapses. In hippocampal slices,these synapses are linked with increased functional excitation in models of temporal lobe epilepsy and epileptic humans. Such injury-driven circuit reorganizations were believed to cause epileptogenesis. Enhanced density of synaptic boutons and axonal strength are observed in layer $\mathrm{V}$ pyramidal neurons of rat neocortex. This came into light when researcher mapped variations in excitatory synaptic connections after injury by employing laser-scanning photostimulation linked with whole-cell patch-clamp recording from layer V cortical neurons. This work has shown that the process of regeneration could lead to cortical connections that possess the property of spatial expansion with functional rigidity. Such connections could serve as substrates for functional recovery following a neuronal damage(Jin, Prince and Huguenard, 2006).

The period after the brain injury relies on Recovery and compensation. Recovery constitutes the restoration of lost function and compensation constitutes to the process of acquiring novel behavior or functions to substitute for those lost following injury. It was reported that flowing an injury, say a stroke, for motor deficits, the total period of recovery would be 30 days. This is for the injuries that may be mild and moderate. For severe injuries, recovery period will be 90 days.

A recovery could be spontaneous, and training induced recovery.

Spontaneous recovery: This kind does not rely on an manual intervetnion.

Here, the injury gets resolved, and there could be a functional variation in a short period following the injury after which the injury becomes focal injury within three months and diffuse injury in six months.

However, three processes have been reported to describe much about the early recovery after injury.

These are Diaschisis reversal: It resolves the issues related to metabolic, and blood flow variations, edema, and as a result there will be a reduced neuronal excitability.

The main outcome is an enhanced activity due to the restoration of intact brain areas that were earlier impaired. So, restoration is an important neural strategy. It could mean that brain circuits that were previously functional are mediating the function after injury. Restoration was observed in the physical and cognitive domains. Kinematics changes: This recovery part is associated with variations in the patterns of movement where compensatory patterns are used. The person could start to complete motor movements in a variety of methods in contrast to that observed before the injury. Such novel movements directly lead to functional outcomes. But compensatory methods could become malfunctional. Another approach is spontaneous recovery where reorganization occurs within and between areas of the nervous system. For instance, several investigators have observed neuroplasticity elements following stroke such as new blood vessel formation, axonal sprouting, neurogenesis and dendritic plasticity(Kerr, Cheng and Jones, 2011), and excitability variations. In brain function, another important change is associated with the triggering of learning networks in the early phase, Here, a plasticity that is identical to the developing brain gets induced. This involves task-learning and motor control networks (Chen, Epstein and Stern, 2010). Therefore, during spontaneous recovery cortical reorganization serves as a compensatory one several neuronal circuits are used following injury than those used before injury. Although a spontaneous recovery takes place without rehabilitation, there is chance that training induced recovery could overlap with it.

Training oriented recovery. After an injury, rehabilitation training could induce plasticity but the period is not definite as spontaneous recovery processes. Here, the recovery could involve compensation that involves the 
specific input of novel neural networks to accomplish earlier tasks. Neuroplasticity gets induced through training process. After injury, adaptive changes indicate the result of new activation patterns which involve the plasticity in surrounding areas of the damaged coexisting networks reorganization or a novel cortical region recruitment.

\section{Recruitment process:-}

Recovery processes that rely on training enable the regions that were non significant for a specific function before injury to become functional after injury. This could necessitate the neural area recruitment from the undamaged hemisphere because it could induce motor movements in a damaged limb that was earlier regulated by the injured motor cortex. Similarly, a neural recruitment could involve a region (right side homologue) that is identical to Broca's area (right side homologue) to enhance the language function if there is damage to the Broca's area. Therefore, such changes through rehabilitation could depend on a constraint induced manual therapy or cognitive tasks completion while utilizing complex hand movements in the opposite hemisphere that facilitates a shift to the undamaged hemisphere. Retraining: This uses providing training to the residual brain regions. This leads to cortical reorganization and a compensation for the functional loss. So, within the damaged hemisphere this activity frequently becomes apparent in the form of reorganization. With regard to motor function, if finger controlling tissue gets lost, other cortical tissue in the vicinity could get reorganized to provide that lost function. As a result, recruitment and retraining necessitate a wide network of neurons. Importantly, it is essential to know the brain properties that offer the recovery mechanisms.

These are brain possess increased redundancy potential. In regions like primary motor cortex,primary auditory cortex, primary visual cortex, and the somatosensory areas, brain exhibits internal redundancy. There are several regions within the primary cortex that react to a given stimuli that may be similar. External redundancy constitutes an identical functionality being processed via several brain regions.

These two redundancies facilitate better integration of information and offer a pathway to enhanced function following the brain damage. Another property is associated with plasticity that is experience dependent. Here, variations in experience or behavior contribute to variations at a neurobiological level (Ashley,2012).

During the recovery, environment also plays important role in contributing to neurological function. This is because as the brain gets adjusted, acquired functions are utilized.This contributes to plasticity changes and a non usage of other regions that does not impact any changes. If the patient is motivated to utilize an undamaged arm instead of the damaged arm, the neuron quantity that gets offered to that region increases in reaction to the usage (Ashley,2012).

This is a counterproductive mechanism because the similar cells could be better put into work for helping the damaged arm as per demand. It is essential to facilitate the best motor patterns while promoting the utilization of the damaged arm in order to avoid the learning of patterns abnormal motor type. Therapists have a role to administer correct level and the time of environmental demand during the recovery process. In response to a direct environmental demand, the existing neural structures are reprogrammed by the brain to involve in a specific function and to forms novel structures to involve in a novel function. A brain could learn a poorly executed sequence and a well-executed motoric movement sequence in a similar fashion.

The acquisition of a specific function in influenced by the interactions between the central nervous system and reinforcement paradigms. Because the brain is programmed to represent a specific task, there is a need of care to guarantee that only the presented stimuli would motivate pro-social or adaptive functions. If not, wrong responses would be learned (Ashley,2012).

In response to pathological conditions like injuries, normal brain vascular system gets stimulated. For instance, in adults, angiogenesis occurs by endothelial progenitor cells (EPCs) and mature endothelial cells. EPCs move to peripheral blood after the brain injury. Following brain injury, an increase in EPC's and CD34 occurs in peripheral blood at 24, reaches maximum at $48 \mathrm{~h}$ and becomes normal at 168 hours.CD34+ cells could be detected soon within 24 hours in the area that surrounds the injury. In addition, EPCs offer endothelial repair and lead to ischemiainduced neovascularization. The activity of EPCs get increased by agents such as erythropoietin, PPAR- $\gamma$ agonists, angiotensin II type 1 receptor blockers, angiotensin-converting enzyme inhibitors, and statins. Researchers described that the angiogenesis could be feasible through the delivery of angiopoietins (Ang), fibroblast growth factor (FGF), and vascular endothelial growth factor (VEGF).In individuals with brain injury, angiogenesis occurs in association with neurogenesis. In the central nervous system (CNS), multi-cellular complexes known as neurovascular units 
(NVUs) are present that possess glial cells, neurons, pericytes, endothelial cells, extracellular matrix proteins and growth factors (Guo \& Lo, 2009).

These components are located very close to the endothelium. In the adult brain, NVUs are considered as the repositories of neural progenitor cells. They harbor recently born neurons that have tight links with the remodeling vascular system. A new vasculature formation could allow the process of coupled neurorestoration like neurogenesis and synaptogenesis that could contribute to enhanced functional recovery ( $\mathrm{Li} \&$ Chopp,2009).

Through the vascular synthesis of Ang1 and stromal-derived factor 1 (SDF1), angiogenesis and neurogenesis are associated. In addition, Ang1 and SDF1 facilitate a post-brain injury migration of neuroblasts and recovery of behavior. In brain disorders like trauma, stroke and infection, an impaired neurovascular coordination was found (Ohab et al., 2006).

It was shown that injured brain could be triggered to facilitate the disruption of the neurovascular coordination in a variety of brain diseases such as infection, stroke, and trauma (Han and Suk,2005).

In mammals, the process of neurogenesis was reported to take place in the olfactory bulb (OB), subventricular zone (SVZ) and subgranular zone (SGZ) of the hippocampus. Here, neuronal cells that are feshl synthesized get initiated from neural stem cells (NSCs). NSCs are the multipotent and self-renewing cells which produce glial cells and neuronal cells. In the hippocampus, dentate gyrus (DG) contains consistently dying granule neurons, and progenitor cells could undergo proliferation compensate for that loss and maintain cell population. Likewise, freshly proliferated cells from SVZ resupply the dead neurons of OB region (Xiong, Mahmood \& Chopp,2010).

Apart from this, resident neural progenitors get stimulated to substitute the acute injury neuronal loss. In the DG of the hippocampus, the newly synthesized neurons were able to form axonal projections to the CA3 region of the brain in the rat models with and without brain injury. So following a neuronal insult, neuroblasts synthesized in the SVZ move to the ischemic boundary zone (IBZ) where angiogenesis takes place (Xiong, Mahmood \& Chopp,2010). During such neuroblast migration, there will be a close association between cerebral vessels and neuroblasts (Thored et al.,2007). In order to independently approach, the ischemic striatum neuroblasts get associated with the microenvironment (Zhang et al., 2009). Following the migration process, SVZ-derived neuroblasts develop into fully functional neurons in the IBZ (Katakowski et al., 2007). After injury, cerebral vessel specific activated endothelial cells produce SDF-1 $\alpha$ to draw the attention of CXCR4 expressing neuroblasts (Hill et al., 2004). CXCR4 is the functional SDF- $1 \alpha$ receptor. Its prevention was found to cancel the phase of neuroblast migration to the IBZ area (Imitola et al, 2004). To this end, pharmacological products like sildenafil and statins were developed that could substantially improve neurogenesis and angiogenesis and enhance recovery during injuries(Zhang ,et al.,2006) . The link between angiogenesis and neurogenesis is so vital such that after brain injury almost all neurorestorative products enhance both these processes to occur (Zhang \& Chopp, 2009). Thus, brain angiogenesis offers the important neurovascular space for neuronal modeling events.

Hence, neuroplasticity after ABI can be considered as an important mechanism for restoring the lost cognitive and behavioral skills in individuals. 


\section{Graphs:-}

(Rapoport and Gogtay,2008)

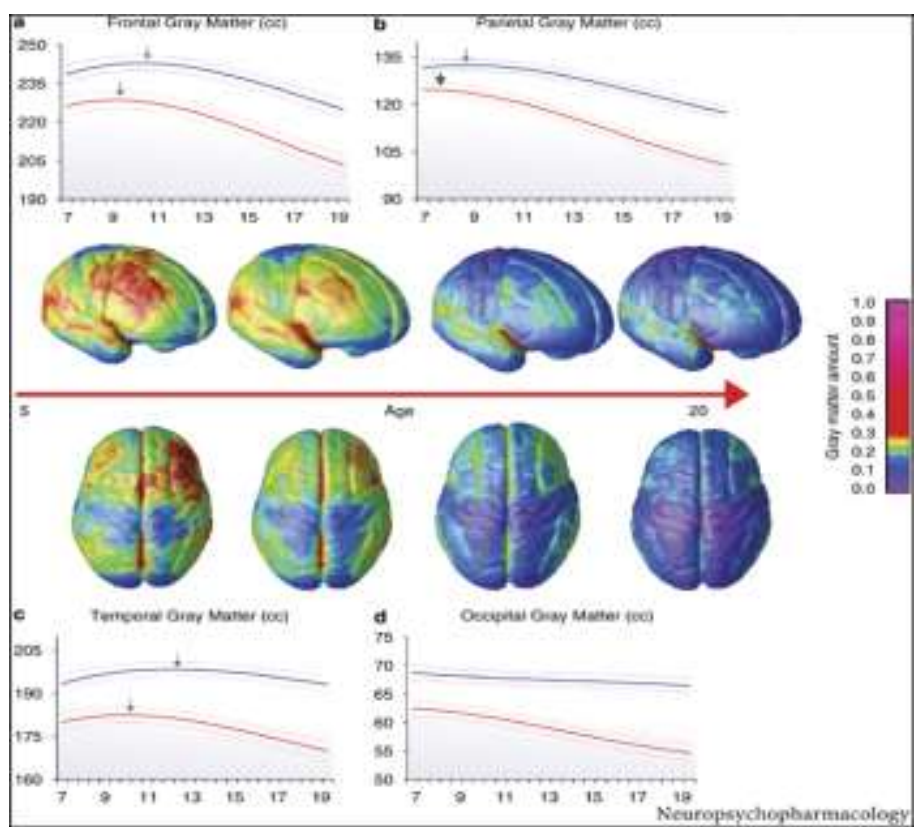

This graph depicts the neuroplasticity changes in the grey matter of brain in individuals aged between 4 and 22 .

\section{References:-}

1. Acquired

brain

injury.,2013.[online]

Available at:http://www.betterhealth.vic.gov.au/bhcv2/bhcarticles.nsf/pages/Acquired_brain_injury?open [Accessed 5 March 2014].

2. Acquired brain injury:ABI manual.,2014.[online] <http://www.acquiredbraininjury.com/abi_manual> [Accessed 5 March 2014]

3. Ashley,M., 2012. Repairing the injured brain.[online] Available at: <http://dana.org/Cerebrum/2012/Repairing_the_Injured_Brain_Why_Proper_Rehabilitation_Is_Essential_to_ Recovering_Function/>

4. Elovic,E., Baerga, E., and Cuccurullo, S.Mechanism and Recovery of Head Injury. In: Cuccurullo S, editor. Physical Medicine and Rehabilitation Board Review. New York: Demos Medical Publishing; 2004. Available from: http://www.ncbi.nlm.nih.gov/books/NBK27256.

5. Guggenmos D.J., Azin, M., Barbay,S., Mahnken, J.D.,Dunham,C., Mohseni, P., and Nudo, R.J., 2013. Restoration of function after brain damage using a neural prosthesis. PNAS, Guo, S. and Lo, E.H.,2009.Review Dysfunctional cell-cell signaling in the neurovascular unit as a paradigm for central nervous system disease.Stroke,40(3 Suppl),pp.S4-7.

6. Guo,Z., Zhang, L., Wu,Z., Chen, Y., Wang, F., and Chen, G.,2014. Head Injury,2011., [online] Available at:http://www.patient.co.uk/doctor/head-injury [Accessed 5 March 2014]

7. Han, H.S. and Suk, K. Review The function and integrity of the neurovascular unit rests upon the integration of the vascular and inflammatory cell systems. Curr Neurovasc Res, 2(5),pp.409-23

8. Hill, W.D, Hess, D.C., Martin-Studdard, A., Carothers, J.J., Zheng, J., Hale, D., Maeda, M., Fagan, S.C., Carroll, J.E. and Conway, S.J. (2004). SDF-1 (CXCL12) is upregulated in the ischemic penumbra following stroke: association with bone marrow cell homing to injury. J Neuropathol Exp Neurol,63(1),pp.84-96.

9. Imitola, J., Raddassi, K., Park, K.I., Mueller, F.J., Nieto, M., Teng, Y.D., Frenkel, D., Li, J., Sidman, R.L., Walsh, C.A. and Snyder, E.Y. (2004). Directed migration of neural stem cells to sites of CNS injury by the stromal cell-derived factor 1alpha/CXC chemokine receptor 4 pathway. Proc Natl Acad Sci U S A, 101(52),pp.18117-18122.

10. In Vivo direct reprogramming of reactive glial cells into functional neurons after brain injury and in an Alzheimer's disease model. Cell Stem Cell,14(2),pp.188-202. 
11. Jin,X., Prince,D.A., and Huguenard,J.R.,2006.Enhanced Excitatory Synaptic Connectivity in Layer V Pyramidal Neurons of Chronically Injured Epileptogenic Neocortex in Rats. J Neuroscience,26(18),pp.48914900.

12. Katakowski, M.,Chen,J., Zhang, Z.G., Santra, M., Wang, Y. and Chopp, M. Stroke-induced subventricular zone proliferation is promoted by tumor necrosis factor-alpha-converting enzyme protease activity. J Cereb Blood Flow Metab,27(4),pp.669-678.

13. Li, Y. and Chopp, M. Marrow stromal cell transplantation in stroke and traumatic brain injury. Neurosci Lett,456(3),pp.120-123.

14. Marasco,P.D., and Kuiken, T.A., 2010.Amputation with Median Nerve Redirection (Targeted Reinnervation) Reactivates Forepaw Barrel Subfield in Rats.J Neuroscience,30(47),pp.16008-16014.

15. Neural plasticity,2014.[online] Available at: $<\mathrm{http}: / /$ www.medterms.com/script/main/art.asp?articlekey=40362> [Accessed 6 March 2014]

16. Novack, $T$ and Bushnik,T., n.d., Understanding TBI: Part 3 - The Recovery Process.[online] Available at:<http://www.msktc.org/tbi/factsheets/Understanding-TBI/The-Recovery-Process-For-Traumatic-BrainInjury $>$ [Accessed 6 March 2014]

17. Novack,T and Bushnik,T., n.d., Understanding TBI: Part 2 - The Recovery Process.[online] Available at:<http://www.msktc.org/tbi/factsheets/Understanding-TBI/The-Recovery-Process-For-Traumatic-BrainInjury $>$

18. Ohab, J.J., Fleming, S., Blesch, A. and Carmichael, S.T., 2006. A neurovascular niche for neurogenesis after stroke. J Neurosci, 26(50),13007-13016.

19. Pekna, M. and Pekny.,2012. The Neurobiology of Brain Injury.[online] Available at:<http://dana.org/Cerebrum/2012/The_Neurobiology_of_Brain_Injury/>[Accessed 7 March 2014]

20. Plasticity,2014. [online] Available at <http://www.oxforddictionaries.com/definition/english/plasticity> [Accessed 4 March 2014]

21. Rapoport,J.L. and Gogtay,N,2008. Brain Neuroplasticity in Healthy, Hyperactive and Psychotic Children: Insights from Neuroimaging. Neuropsychopharmacology, 33, pp. 181-197.

22. Ritcher,J.D. and Klann., 2009.Making synaptic plasticity and memory last: mechanisms of translational regulation.Genes \& Dev, 23,pp.1-11

23. Ryest, H.,2014. Neuroplasticity after aquired-brain-injury <https://www.rainbowrehab.com/neuroplasticityaquired-brain-injury/> [Accessed 7 March 2014].

24. Sabel,B.A.,Kenkel,S. and Kasten,E.,2005.Vision restoration therapy.Br J Ophthalmol,89(5),pp.522-524.

25. Stern,W.E., 1964. Restoration of function after brain injury.Calif Med,101 (1),pp.70-71

26. Synaptic plasticity,2013.[online] Available at:<http://www.qiagen.com/products/genes\%20and\%20pathways/complete\%20biology\%20list/synaptic\%20pla sticity/> [Accessed 7 March 2014]

27. The brain injury,.n.d.[online] Available at: <http://tbirecovery.org/Therapies.html> [Accessed 7 March 2014]

28. Thored, P., Wood, J., Arvidsson, A., Cammenga, J., Kokaia, Z. and Lindvall, O.Long-term neuroblast migration along blood vessels in an area with transient angiogenesis and increased vascularization after stroke.Stroke,38(11),pp.3032-9

29. Wilson, P.H. Thomas, P., Shum, D,Duckworth, J, Gugliemetti, M., Rudolph, H., Mumford, N., and Eldridge, R. A multilevel model for movement rehabilitation in Traumatic Brain Injury (TBI) using Virtual Environments., 2006. Virtual Rehabilitation. [online] Available at:<http://www98.griffith.edu.au/dspace/bitstream/handle/10072/13286/40447.PDF?sequence=1>[Accessed 7 March 2014]

30. Xiong,Y., Mahmood,A. and Chopp, M. Angiogenesis, neurogenesis and brain recovery of function following injury. Curr Opin Investig Drugs, 11(3)pp.298-308.

31. Zhang, R.L., Zhang, Z., Zhang, L., Wang, Y, Zhang, C. and Chopp, M.,2006. Delayed treatment with sildenafil enhances neurogenesis and improves functional recovery in aged rats after focal cerebral ischemia. $\mathrm{J}$ Neurosci Res,83(7),pp.1213-9.

32. Zhang, Z.G. and Chopp, M., 2009.Review Neurorestorative therapies for stroke: underlying mechanisms and translation to the clinic. Lancet Neurol,8(5),pp.491-500.

33. Zhang, R.L., Chopp, M., Gregg, S.R., Toh, Y., Roberts, C., Letourneau, Y., Buller, B., Jia, L., Nejad Davarani,S,

34. Zhang, Z.G. Patterns and dynamics of subventricular zone neuroblast migration in the ischemic striatum of the adult mouse.J Cereb Blood Flow Metab,29(7),pp.1240-50.(Zhang,et al., 2006). 